\title{
Texture Features Based on Area Ternary Pattern for Classification Collagen Second Harmonic Generation Images of Skin Scar
}

\author{
Gaoqiang Liu, Mingyu Liu, Jichun Li, Encai Zhang, Kun Zhang, and Guannan Chen ${ }^{a}$ \\ Key Laboratory of Optoelectronic Science and Technology for Medicine, Ministry of Education \\ Fujian Normal University \\ Fuzhou, 350007, China
}

\begin{abstract}
We proposed a method that based on area ternary pattern (ATP) operator to extract texture features of collagen second harmonic generation (SHG) images of skin scar. The proposed method includes texture feature extraction in collagen SHG images of skin scar, and classification with a support vector machine. By comparing with other texture feature extractive methods relating to the accuracy with specificity and sensibility, the method was demonstrated to obtain higher accuracy and better performance. It is credible that the proposed method improves clinical diagnosis of scar types and educes the development of new curative methods for dermatology and plastic surgery.
\end{abstract}

\section{Introduction}

Most wound on the skin healings result in scars especially after deep dermal injuries, mainly including normal and pathologic scars.[1] What scar may bring about pain, deformity, or limiting joint mobility is an inconvenient presence. We need distinguish different methods to aim at specific scars because different scars need to be treated in different ways. Nowadays, clinical examination and biopsy, which is complicated, are the main way to diagnose the types of scar.[2] Thus, clinicians and patients desire a simple and effective method for characterizing scar tissues. Image analysis of collagen fibers permit us to achieve the aim, since it has special and unique characteristics. What texture analysis about quantitative measures are hot research methods now can provide a novel technique for quantitative analysis.

The virtue of texture feature that is a kind of visual characteristics reflects the homogeneity of an image rather than depending on colors and brightness.[3] Appearance, arrangement and structure of image belong to image texture. Nowadays, there is not widely accepted texture extraction method that is fit for all types of medical images, because the texture information of image is complex.[4] Therefore, researching more accurate texture analysis method is instant and difficult task in current medical image processing researches.

In this paper, we discussed the texture feature extraction of collagen SHG images in human skin scars. The new local operator combined with line-likeness and phase congruency to represent texture information of medical images. In addition, we make the comparisons using these texture parameters and classification of scar images to evidence the effectiveness of proposed method.

\section{Materials and methods}

\subsection{Images acquisition}

\footnotetext{
$\overline{{ }^{a} \text { Corresponding author:edado@fjnu.edu.cn }}$
} 
We obtained these ripen skin scar specimens from our patients undergoing cesarean section located at the abdomen. Informed consent was achieved from every patient who participated in the study.

The normal and hypertrophic scar samples were collected during surgery at the abdomen from 4 subjects aged 29 to 55 years old. The samples were snap-frozen in liquid nitrogen $\left(-196{ }^{\circ} \mathrm{C}\right)$ to store right now after being severed from patients. We chose the center of the severed tissue as scar region for examination. SHG images were achieved using a commercial laser scanning microscopic imaging system equipped with a mode-locked femtosecond Ti: sapphire laser operating at $810 \mathrm{~nm}$. The acquisition of the SHG image with $512 \times 513$ pixels was about $1.57 \mathrm{~s}$. In this study, one SHG image of collagen was acquired from each normal scar and skin tissue section.

\subsection{Procedure of the proposed method}

Fig. 1 shows the procedure of the proposed method, which included four steps as follows. Firstly, we acquired the original collagen SHG image from the surgery at the abdomen. Secondly, using area ternary pattern operator in original image acquire ATP image. Thirdly, The texture features including line-likeness and phase congruency of ATP image were extracted. At last, the texture features were classified by support vector machine (SVM). The detailed description could be found in the following sections.

\subsection{Texture extraction}

LTP operator has many advantages including robustness to illumination, rotation invariance and computational simplicity.[5] However, domain of LTP operator is limited in $3 \times 3$ neighborhoods, so texture information Reserved is restricted.

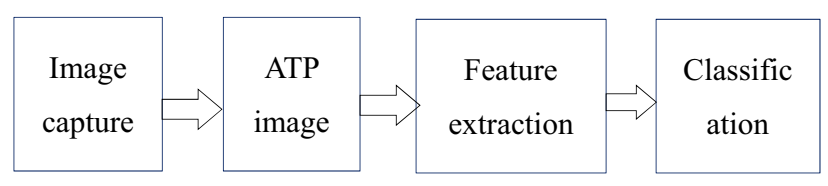

Figure 1. Procedure of the proposed method

We proposed a new local operator area ternary pattern (ATP) that is inspired from LTP operator. Extracting the line-likeness and phase congruency of ATP image is expedient to quantify texture features within the scar collagen SHG images. ATP is devised to inherited the advantages and extend function neighborhood to enhance effectiveness. Fig. 2 illuminates the procedure of ATP operator. The function neighborhood of ATP's center pixel $g_{c}$ that is gray value of original image is $k \times k$ neighborhood, where $k=2 x+1, x \in[1,2,3,4]$. As for a center pixel $g_{c}$, we could achieve the mean $g_{k}$ of each $k \times k$ neighborhood. For decreasing the computational complex, we assume binary code instead of ternary code and disintegrate it into upper and lower patterns. In the end, It will be a pixel value in ATP image after upper and lower patterns perform a bitwise OR operation in decimal.The ATP code could be acquired in Equation 1.

$$
A T P=\sum_{x=1}^{4} s\left(g_{k}-g_{c}\right) 2^{p}, s(x)= \begin{cases}1, & x>t \\ 0, & -t \leq x \leq t, k=2 x+1 \\ -1, & x<-t\end{cases}
$$

Where $t$ is theshold that is the mean of $g_{k}$. In addition, for maintaining the uniform of code, we selected the ATP's

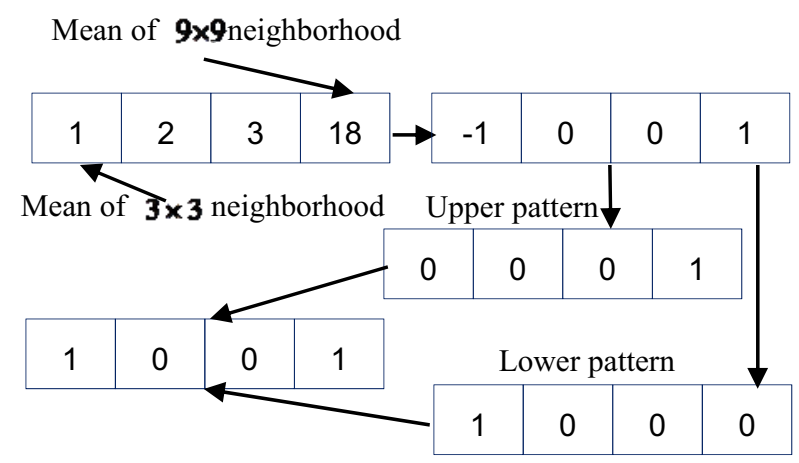

Figure 2. The procedure of ATP operator (value of center pixel is 8)

code that only consist of at most one $0-1$ and one 1-0 conversion when perceived as a rounded bit string. Due to the uncertainty of binary code of uniform pattern, we could not ensure correspondent decimal. The minimum value is defined as rotation invariance pattern to solve the problem.

Line-likeness is a visual perception of textural features to represent lines, which is regarded as the number of equal for the direction of target edge and neighbor edge.[6] As a measure of line-likeness, co-occurrences in the same direction are weighted by +1 and vertical direction by -1 . The line-likeness was calculated as follows: 


$$
F_{\text {lin }}=\sum_{i}^{n} \sum_{j}^{n} P(i, j) \cos \left[(i-j) \frac{2 \pi}{n}\right] / \sum_{i}^{n} \sum_{j}^{n} P(i, j)
$$

where $P(i, j)$ is the $n \times n$ local direction co-occurrence matrix of points at distance. The distance function $d_{4}$ was using four in our experiments.

Phase congruency (PC) is a dimensionless measure for the importance of a structure individually of the signal amplitude.[7] The relevant technique to calculate PC used in this paper is effective to combine with ATP. The phase congruency idea is rooted in the supposition that features are observed in image points, where Fourier constituents of the signal are maximally in phase. What the PC is expressed as the summation over orientation $\mathrm{o}$ and scale $\mathrm{n}$ was calculated as follows:

$P C(x, y)=\frac{\sum_{o} \sum_{n} W(x)\left\lfloor A_{n o}(x, y) \Delta \phi_{n o}(x, y)-T\right\rfloor}{\sum_{o} \sum_{n} A_{n o}(x, y)+\varepsilon}$

$$
\Delta_{n o}(x, y)=\cos \left(\phi_{n o}(x, y)-\bar{\phi}(x, y)\right)-\left|\sin \left(\phi_{n o}(x, y)-\bar{\phi}(x, y)\right)\right|
$$

$$
W(x)=\frac{1}{1+e^{r(c-s(x))}}
$$

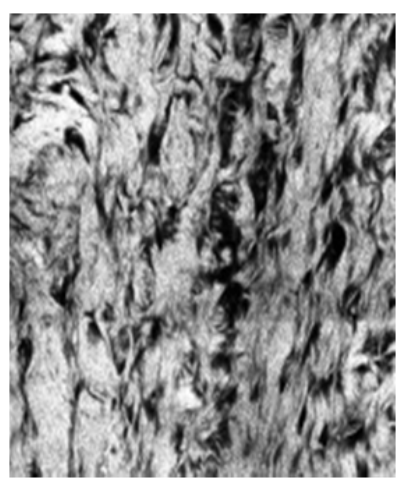

(a)

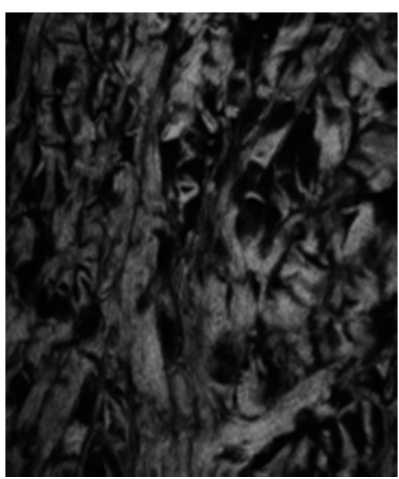

(b)
Figure 3. Collagen SHG images in human (a) normal and (b) hypertrophic scar. Images are $512 \times 513$ pixels

$$
s(x)=\frac{1}{N}\left(\frac{\sum_{n} A_{n}(x)}{A_{\max }(x)+\varepsilon}\right)
$$

where \lfloor\rfloor denotes that the enclosed quantity is equal to itself when its value is positive, otherwise it is zero. $T$ is a additional item for noise and $\varepsilon$ is a compensate item for avoiding division by zero. $\Delta \phi_{n o}(x, y)$ is a sensitive measure of phase deviation. $\phi_{n o}(x, y)$ is phase angle and $\overline{\phi_{o}}(x, y)$ is the mean phase angle at orientation o. $W(x)$ is a weighting function including threshold $c$ of filter response and control factor $r$ of sharpness of the threshold. $s(x)$ is frequency response spread function.

\subsection{SVM}

Support Vector Machine (SVM) is a mighty tool to binary classification in the field of pattern recognition and machine vision.[8] Because SVM have powerful classification performance and train samples speedily, we focused on SVM in this work. The SVM algorithm that is stemmed from the notion of the structural risk minimization is outstanding to learn non-linear or linear class boundaries.

The SVM algorithm functions as input labelled data from two groups, and generates new labelled or unlabeled data by an outputs model for classifying. As with many pattern methods, the classifier has two main processes that is training and testing. In the course of training, the training set consists of training data and label. The input data is applied to shine upon higher dimensional space in SVM by radial basis function (RBF) kernel. A hyper plane linear classifier is utilized in the higher dimensional space by pattern vectors. The RBF is shown as follows:

$$
k\left(x_{i}, x_{j}\right)=\exp \left(-\gamma\left\|x_{i}-x_{j}\right\|^{2}\right), \gamma>0
$$

where $x_{i}$ and $x_{j}$ are training vectors, $\gamma$ is parameter of kernel.

\section{Results and discussion}

The collagen SHG images in human normal and hypertrophic scar tissues are revealed in Fig. 3. It is difficult to distinguish normal scar (see Fig. 3(a)) and hypertrophic scar (see Fig. 3(b)) in unaided eye because of similarity of fibroblasts and deposition of collagen fibers. So as to quantitatively depict the scars, we extracted texture features in normal and hypertrophic scar collagen SHG images. The proposed method that extracted the line-likeness and phase congruency of ATP images was suggested to describe the texture features in normal and hypertrophic scar collagen SHG images. 
We compared our algorithm based on ATP operator with LTP operator and gray level co-occurrence matrix (GLCM) to reflect the validity. GLCM, which is extensively used for texture feature extraction, is based on gray-level statistical models in an image. GLCM could provide four texture features including homogeneity, entropy, contrast and correlation in grayscale images. Comparison of LTP and

Table 1. Accuracy rates of different texture feature

\begin{tabular}{|c|c|c|c|}
\hline Type & GLCM(\%) & LTP(\%) & ATP(\%) \\
\hline normal & 66.67 & 46.67 & 80.00 \\
\hline hypertrophic & 80.00 & 86.67 & 86.67 \\
\hline overall & 73.33 & 66.67 & 83.33 \\
\hline
\end{tabular}

GLCM with ATP is shown in Table 1. It is obvious that accuracy rate required by our algorithm based on ATP is significantly better than other methods. The recognition effects of LTP operator and GLCM separately are worse in relation to ATP.

We evaluated the performance of proposed method that line-likeness and phase congruency of ATP image are extracted using leaving one out. From the result of experiment, ATP operator have higher sensibility and lower specificity then other methods. In summary, we believe the ATP operator provide a effective method to achieve a relatively good performance for scar diagnosis.

\section{Conclusion}

In this work, a new method that extracted the line-likeness and phase congruency of ATP image in collagen SHG images from human normal and hypertrophic scar was proposed. Extracting the line-likeness and phase congruency of ATP image was applied to classify the images by SVM classifier. The proposed method about ATP operator was demonstrated to have highly advantage after comparing with other texture feature extraction methods. The proposed method belongs to image technology that is a noninvasive method for the scar diagnosis, which could be aroused by the development of new therapies and has highly application potential in the studies of some other domain.

We are focusing on more effective approaches to extract heterogeneous texture features of human scars. We believe the higher accuracy classifier of texture features will emerge in the near future.

\section{Acknowledgement}

This work is supported by the National Natural Science Foundation of China (Nos. 81101110), the Science and Technology Project of Fujian Province (Nos.2015J01300), and Fujian Normal University foundation for excellent young teachers (No. fjsdjk2012058).

\section{References}

1. X. Zhu, S. Zhuo, L. Zheng, K. Lu, X. Jiang, J. Chen, et al., J BIOPHOTONICS, vol. 3(2010).

2. K. Tan, D. McGrouther, A. Day, C. Milner, and A. Bayat, J EUR ACAD DERMATOL, vol. 25(2011).

3. B. Li and M. Q.-H. Meng, IMAGE VISION COMPUT, vol. 27(2009).

4. R. Ambekar, T.-Y. Lau, M. Walsh, R. Bhargava, and K. C. Toussaint, BIOMED OPT EXPRESS, vol. 3( 2012).

5. X. Tan and B. Triggs, Analysis and Modeling of Faces and Gestures(ed: Springer, 2007)

6. T. Majtner and D. Svoboda, 3D Imaging, Modeling, Processing, Visualization and Transmission (3DIMPVT, 2012)

7. L. Zhang, L. Zhang, D. Zhang, and Z. Guo, PATTERN RECOGN, vol. 45(2012).

8. M. Pal and G. M. Foody, IEEE T GEOSCI REMOTE, vol. 48(2010). 